\section{Evaluación de MALDI-TOF MS para la identificación de levaduras patógenas oportunistas de muestras clínicas}

\author{
Alexandro Bonifaz', Fernando Montelongo-Martínez', \\ Javier Araiza', Gloria María González², Rogelio \\ Treviño-Rangel2, Alejandro Flores-Garduño ${ }^{3}$, \\ Alejandro Camacho-Cruz ${ }^{4}$ y Andrés Tirado-Sánchez
}

Evaluation of MALDI-TOF MS for the identification of opportunistic pathogenic yeasts of clinical samples

MALDI-TOF MS mass spectrometry is a rapid and straightforward technique to identify microorganisms by protein analysis. The study was performed in 304 yeast isolates from superficial and deep mycoses, in order to compare three methods: conventional (biochemical and morphological), MALDI-TOF MS, and polymerase chain reaction (PCR, reference). We included 24 species with predominance of Candida spp and Cryptococcus spp. The identification by conventional methods was 258/304 strains, while by MALDI-TOF MS was: $277 / 304$ strains $(84.8 \%$ versus $91.2 \%, P=$ not significant). The Kappa coefficient comparing MALDI-TOF-MS with PCR reported excellent concordance (0.99). The sensitivity and specificity of MALDI-TOF MS for the diagnosis of opportunistic pathogenic yeasts of clinical samples were $94.6 \%$ and $99 \%$ respectively. MALDI-TOF MS is a simple, fast and reliable tool for pathogenic yeasts.

Keywords: MALDI-TOF MS, Mass Spectrometry, Opportunistic yeast, Short extraction, Protein Mass Fingerprinting.

Palabras clave: MALDI-TOF MS; Espectrometría de masas; levaduras oportunistas; extracción corta; huella dactilar de masas.

\section{Introducción}

$\mathrm{L}$ as micosis causadas por levaduras patógenas oportunistas, son frecuentes en pacientes con diversos estados de inmunosupresión y en inmunocompetentes; su identificación es indispensable para la selección del tratamiento adecuado. La mayoría de ellas están incluidas en dos géneros: Candida y Cryptococcus, y en menor proporción: Trichosporon, Geotrichum entre otras ${ }^{1,2}$. La identificación se hace mediante técnicas

'Laboratorio de Micología. Servicio de Dermatología. Hospital General de México. “Dr. Eduardo Liceaga".

²Departamento de Microbiología, Facultad de Medicina, Universidad Autónoma Nuevo León (UANL), Monterrey, México.

${ }^{3}$ Laboratorio de Bacteriología. Servicio de Laboratorios Centrales. Hospital General de México, "Dr. Eduardo Liceaga".

${ }^{4}$ Cepario de la Facultad de Química. Universidad Nacional Autónoma de México (UNAM), Ciudad de México.

Conflictos de interés: Los autores declaran no tener ningún conflicto de interés.

Financiamiento: Para la realización de este trabajo no se contó con ningún soporte económico. Informamos que este trabajo fue sometido y aceptado por el comité científico y de ética de nuestro hospital, quedando registrado con el número: DI/15/109/03/30.

Recibido: 9 de diciembre de 2018 / Aceptado: 15 de octubre de 2019

Correspondencia a: convencionales (bioquímicas, morfológicas) y moleculares (RPC), y cada una de ellas varía en sensibilidad, costo y disponibilidad ${ }^{3}$. MALDI-TOF MS (Matrix Assisted Laser Desorption Ionization Time of Flight Mass Spectrometry), es un método proteómico de ionización suave que fragmenta proteínas ribosomales, para obtener un espectro de masas específico para cada taxón fúngico, conocido como "huella dactilar de masas" (Protein Mass Fingerprinting $)^{4-8}$. El objetivo de este estudio fue la identificación de levaduras aisladas de micosis mediante MALDI-TOF MS y compararla con las técnicas convencionales, teniendo como referencia a la RPC (estándar de oro).

\section{Método}

Se aislaron y estudiaron 304 aislados de levaduras de pacientes del Hospital General de México "Dr. Eduardo Liceaga” de muestras procedentes de infecciones superficiales y profundas. Los primo-cultivos se realizaron en agar dextrosa Sabouraud con cloranfenicol, CHROMagar-Candida ${ }^{\circledR}$ y agar alpiste negro. Se realizaron tres tipos de identificación: 1) Método convencional (MC): pruebas micológicas y asimilación de carbohidratos $\left(\text { VITEK }^{\circledR} 2 \text { YST ID-card }\right)^{9,10} ; 2$ ) Identificación proteómica por MALDI-TOF MS: las levaduras fueron sembradas en agar papa dextrosa e incubados a $28^{\circ} \mathrm{C}$ y con no más de $48 \mathrm{~h}$. Las muestras fueron analizadas con el equipo comercial MALDI-TOF Vitek-MS ${ }^{\circledR}$, utilizando la metodología de extracción corta en placa con $0.5 \mu \mathrm{L}$ de ácido fórmico al $25 \%$, para romper la pared celular y $1,0 \mu \mathrm{L}$ de la solución $3,1 \% \mathrm{v} / \mathrm{v}$ de ácido $\alpha$-ciano4-hidroxicinámico para cristalizar las proteínas extraídas ${ }^{4,6-8,10}$, se tomaron en cuenta las identificaciones con alto valor de concordancia (>98\%); 3). Identificación por biología molecular: se extrajo el ADN genómico de las levaduras mediante el método de fenol-cloroformo ${ }^{11}$. El material genético resultante se utilizó como templado para la posterior amplificación del espaciador Transcripcional Interno (ITS), la cual es una región no codificante del ARNr, utilizando los iniciadores ITS5 e ITS4 ${ }^{12}$. Una vez verificada la presencia de los amplicones, éstos se purificaron mediante kit comercial (Wizard, Promega) y se secuenciaron por el método de Sanger en el analizador genético ABI 3130 (Applied Biosystems) siguiendo las recomendaciones del fabricante. Las secuencias obtenidas se compararon con los depósitos del GenBank utilizando la plataforma bioinformática BLASTn Data-base (National Center for Biotechnology Information). Dado el elevado poder discriminatorio que brinda esta herramienta molecular, se consideró como el método de referencia para la identificación definitiva de las levaduras incluidas en el presente estudio ${ }^{3,13}$.

\section{Resultados}

En la Tabla 1, se presentan los sitios de aislamiento de las levaduras incorporadas al estudio. Los datos referentes a la identificación mediante las tres técnicas del estudio se presentan en la Tabla 2. No observamos diferencias en el diagnóstico de Candida albicans, especies no-albicans y otros hongos levaduriformes (Tabla 3 ).

El coeficiente Kappa entre la técnica de identificación convencional y el sistema MALDI-TOF-MS fue de 0,506, que según la evaluación de este índice mediante la escala Landis y Koch, indica una buena concordancia. ${ }^{14}$ Si tomamos este coeficiente Kappa comparando MALDI-TOF-MS con el método de referencia (RPC), MALDI-TOF-MS mantiene una excelente concordancia $(0,99)$. 


\begin{tabular}{|c|c|}
\hline Levadura & Cantidad cepa / muestra biológica \\
\hline Candida albicans & $\begin{array}{l}26 \text { / LBA } \\
12 \text { / Orina } \\
9 \text { / Boca } \\
6 \text { / Piel } \\
4 \text { / Uñas }\end{array}$ \\
\hline Candida dubliniensis & $1 /$ LBA \\
\hline Candida glabrata & $\begin{array}{l}21 \text { / Orina } \\
14 \text { / Boca } \\
8 \text { / LBA } \\
1 \text { / Líquido de diálisis }\end{array}$ \\
\hline Candida guilliermondii & $\begin{array}{l}1 / \text { uñas } \\
1 / \text { piel }\end{array}$ \\
\hline Candida haemuloni & $1 /$ LBA \\
\hline Candida intermedia & 1 / Orina \\
\hline Candida krusei & $\begin{array}{l}8 \text { / Orina } \\
3 \text { / Mucosa nasal } \\
2 \text { / Boca } \\
1 \text { / LBA } \\
1 \text { / Piel } \\
1 \text { / Esputo }\end{array}$ \\
\hline Candida lambica & 2 / Sangre \\
\hline Candida lipolytica & $\begin{array}{l}2 \text { / Piel } \\
1 \text { / Boca } \\
1 \text { / Sangre }\end{array}$ \\
\hline Candida lusitaniae & $\begin{array}{l}2 \text { / Esputo } \\
1 / \text { LBA }\end{array}$ \\
\hline Candida metapsilosis & $5 /$ LBA \\
\hline Candida orthopsilosis & $\begin{array}{l}6 \text { / Uña de mano } \\
2 \text { / Piel }\end{array}$ \\
\hline Candida parapsilosis & $\begin{array}{l}18 \text { / Uñas. } \\
10 \text { / LBA } \\
3 \text { / Oído } \\
5 \text { / Orina } \\
4 \text { / Boca } \\
1 \text { / Piel } \\
2 \text { / Ojo }\end{array}$ \\
\hline Candida tropicalis & $\begin{array}{c}27 \text { / LBA } \\
10 \text { / Orina } \\
3 \text { / uñas }\end{array}$ \\
\hline Cryptococcus albidus & $1 / \mathrm{LCR}$ \\
\hline Cryptococcus Gatti & $2 / L C R$ \\
\hline Cryptococcus neoformans & $35 /$ LCR \\
\hline Geotrichum candidum & 1 / Piel \\
\hline Rhodotorula mucilaginosa & $\begin{array}{l}1 \text { / Sangre } \\
1 \text { / Esputo }\end{array}$ \\
\hline Saprochaete capitata & $1 /$ Boca \\
\hline Trichosporon asahii & $\begin{array}{l}3 \text { / LBA } \\
2 \text { / Esputo } \\
2 \text { / Sangre } \\
2 \text { / Pelo (piedra) }\end{array}$ \\
\hline Trichosporon dermatis & $\begin{array}{l}4 \text { / Piel } \\
2 \text { / Uñas }\end{array}$ \\
\hline Trichosporon inkin & 7 / Pelo \\
\hline Prototheca wickerhamii & 1 / Líquido de ascitis \\
\hline
\end{tabular}

\begin{tabular}{|c|c|c|c|}
\hline $\begin{array}{l}\text { Levaduras } \\
\text { Especies }\end{array}$ & $\begin{array}{c}\text { RPC } \\
\text { (Referencia) }\end{array}$ & $\begin{array}{l}\text { Métodos } \\
\text { Método } \\
\text { convencional }\end{array}$ & $\begin{array}{l}\text { MALDI- } \\
\text { TOF MS }\end{array}$ \\
\hline Candida albicans & 57 & 52 & 56 \\
\hline Candida dubliniensis & 1 & 1 & 1 \\
\hline Candida glabrata & 44 & 42 & 44 \\
\hline Candida guilliermondii & 2 & 1 & 1 \\
\hline Candida haemuloni & 1 & 1 & 1 \\
\hline Candida intermedia & 1 & 0 & 1 \\
\hline Candida krusei & 16 & 15 & 16 \\
\hline Candida lambica & 2 & 2 & 2 \\
\hline Candida lipolytica & 4 & 1 & 3 \\
\hline Candida lusitaniae & 3 & 2 & 3 \\
\hline Candida metapsilosis & 5 & 0 & 0 \\
\hline Candida orthopsilosis & 8 & 0 & 0 \\
\hline Candida parapsilosis & 43 & 40 & 43 \\
\hline Candida tropicalis & 40 & 36 & 38 \\
\hline Cryptococcus albidus & 1 & 1 & 1 \\
\hline Cryptococcus gattii & 2 & 2 & 2 \\
\hline Cryptococcus neoformans & 35 & 35 & 35 \\
\hline Geotrichum candidum & 1 & 1 & 1 \\
\hline Rhodotorula mucilaginosa & 2 & 2 & 2 \\
\hline Saprochaete capitata & 1 & 1 & 1 \\
\hline Trichosporon asahii & 9 & 7 & 9 \\
\hline Trichosporon dermatis & 6 & 0 & 0 \\
\hline Trichosporon inkin & 7 & 6 & 7 \\
\hline $\begin{array}{l}\text { Prototheca wickerhamii } \\
\text { (Alga aclorofilica patógena) }\end{array}$ & 1 & 1 & 1 \\
\hline $\begin{array}{l}\text { Número total: } 304 \\
\text { \% Identificación }\end{array}$ & $\begin{array}{c}292 \\
(100)\end{array}$ & $\begin{array}{c}249 / 292 \\
(85,3)\end{array}$ & $\begin{array}{c}268 / 292 \\
(91,8)\end{array}$ \\
\hline
\end{tabular}

En cuanto a la sensibilidad y la especificidad de MALDI-TOF-MS para levaduras, se obtuvo sensibilidad de 94,6\% (IC95\%: 86,2-96,4\%), y especificidad de 99\% (IC95\%: 92,9-99,3\%). La sensibilidad y especificidad de MALDI-TOF-MS para Candida albicans alcanzó un 98,2\% (IC95\%: 92,7-96,2\%) y 99\% (IC95\%: 92,5-99,8\%): respectivamente. Por otro lado, la sensibilidad y la especificidad para especies no Candida albicans fue de 89,7\% (IC95\%: 82,9-95,3\%), y 99\% (IC95\%: 95,6-99,2\%); respectivamente. La sensibilidad y la especificidad para otros hongos levaduriformes fue de 97,3\% (IC95\%: 88,9-98,3\%) y 99\% (IC95\%: 93,6$99,4 \%$ ); respectivamente. 


\begin{tabular}{|lcccc|}
\hline \multicolumn{2}{|l|}{ Tabla 3. Reportes de identificación mediante RPC, cultivo y MALDI-TOF-MS } & & \\
\hline Especies & RPC Referencia (Estándar de oro) & Identificación convencional & MALDI-TOF-MS & Valor de $\mathbf{p}$ \\
Candida albicans & 57 & 52 & 56 & NS \\
Especies de Candida no-albicans & 170 & 141 & 153 & NS \\
Otros hongos levaduriformes & 65 & 56 & 59 & NS \\
\hline NS $=$ No significativo. & & & \\
\hline
\end{tabular}

\section{Discusión}

La identificación de las levaduras en la mayoría de los laboratorios en los países en vías de desarrollo todavía se realizan por métodos convencionales. En una investigación reciente de Falci y Pascualotto, sobre la capacidad diagnóstica micológica en Latinoamérica, y el Caribe indican que sólo $19 \%$ de los laboratorios identifican por técnica proteómica, contra $71 \%$ por métodos bioquímicos estandarizados ${ }^{15}$. Su inconveniente es el costo y tiempo que consumen ${ }^{2,3,9}$. La identificación molecular (RPC) es la más precisa y se considera de referencia ${ }^{3,9}$. MALDI-TOF MS es una técnica útil y rápida para la identificación de diversos microorganismos ${ }^{4}$. Algunos autores han discutido sobre sus ventajas e inconvenientes al compararla con otras metodologías, considerándola más sensible que los métodos bioquímicos automatizados ${ }^{4,7}$. Otros estudios indican que los resultados con la técnica MALDI-TOF MS son equivalentes a los obtenidos por técnicas de biología molecular hasta en un $95 \%{ }^{9}$.

En cuanto a los resultados de identificación, estos son similares a los reportados por Relloso y cols ${ }^{16}$. Haciendo un análisis por grupo de levaduras, se puede interpretar que: del género Candida, se incluyeron 14 especies, la mayoría se identificaron de forma correcta, con excepción de las especies del complejo Candida-parapsilosis, es decir, C. orthopsilosis y C. metapsilosis, mientras que si identificó a C. parapsilosis $(s s)^{4-9,17}$. Debido a la alerta mundial de infecciones por Candida auris, con este sistema ya se ha reportado su identificación distinguiéndola de Candida haemulonii y de Saccharomyces cerevisiae ${ }^{18}$.

Con respecto a las tres especies de Cryptococcus (C. neoformans, C. gattii y C. albidus), fueron identificadas por ambos métodos. En las especies del género Trichosporon, no se pudo identificar a T. dermatis. El resto de levaduras, G. candidum, S. capitata, R. mucilaginosa y Prototheca wickerhamii, (alga aclorofílica patógena indistinguible de las levaduras), fueron identificadas correctamente. Los resultados fueron similares a los reportados en varios estudios comparativos ${ }^{4-9,10,19,20}$.

En nuestra investigación, MALDI-TOF MS tuvo una buena correlación con las técnicas convencionales de diagnóstico, con una sensibilidad y especificidad del 94,6 y 99\%; respectivamente. Lo anterior está en concordancia con otros estudios ${ }^{7,8,10,15,17,18}$; aunque en nuestro estudio se realizó con una mayor diversidad de especies.

Nuestra interpretación de que algunas levaduras no fueron identificadas, es que éstas son especies que cambian al envejecer sus estructuras de reproducción anamorfa, es decir, son cepas que tienen la capacidad de formar abundantes seudohifas, hifas y/o artroconidios (este último para Trichosporon $)^{17}$, y por lo tanto, presentar más de un estadio de maduración. Este efecto puede alterar la reproducibilidad del espectro y evitar una identificación correctat ${ }^{1,3,7}$.
MALDI-TOF MS es un sistema que tiene la ventaja de la rapidez, y conforme se actualicen sus series de bases se incrementará su sensibilidad/ especificidad y que la mayoría de los equipos comerciales sean compatibles. En la actualidad hay nuevas variantes de esta tecnología que estarán incorporadas en un futuro cercano como son el immuno-MALDI-TOF y el MALDI-TOF IMS (imaging mass spectrometry), el primero con una fusión con las técnicas inmunológicas y el segundo otorgando dimensión de masas ${ }^{10,17,19}$, que sin duda serán más precisas.

\section{Resumen}

La espectrometría de masas MALDI-TOF MS es una técnica rápida y sencilla para identificar microorganismos por análisis proteico. Se estudiaron 304 aislados de levaduras procedentes de micosis superficiales y profundas, con el objetivo de comparar tres métodos: convencional (bioquímico y morfológico), MALDI-TOF MS, y reacción en cadena de la polimerasa (RPC, método de referencia). Se estudiaron 24 especies con predominio de Candida spp y Cryptococcus spp. La identificación por método convencional fue de 258/304 cepas, mientras que por MALDI-TOF MS fue de: $277 / 304$ cepas (84,8 versus $91,2 \%, p=$ no significativo). El coeficiente Kappa entre el MALDI-TOF-MS y la RPC reportó una excelente concordancia $(0,99)$. La sensibilidad y la especificidad de MALDI-TOFMS para la identificación de levaduras patógenas oportunistas de muestras clínicas fueron de 94,6\% y 99\%; respectivamente. MALDI-TOF MS demostró ser una herramienta de alta precisión para la identificación de levaduras patógenas.

\section{Referencias bibliográficas}

1.- Hazen K C. New and emerging yeast pathogens. Clin Microbiol Rev 1995; 8: 462-78.

2.- Vázquez-González D, Perusquía-Ortiz A M, Hundeiker M, Bonifaz A. Opportunistic yeast infections: candidiasis, cryptococcosis, trichosporonosis and geotrichosis. J Dtsch Dermatol Ges 2013; 11: 381-93

3.- Pincus D H, Orenga S, Chatellier S. Yeast identification--past, present, and future methods. Med Mycol 2007; 45: 97-121.

4.- Chalupová J, Raus M, Sedlářová M, Sebela M. Identification of fungal microorganisms by MALDI-TOF mass spectrometry. Biotechnol Adv 2014; 32: 230-41. doi: 10.1016/j.biotechadv.2013.11.002.

5.- Clark A E, Kaleta E J, Arora A, Wolk D M. Matrix-assisted laser desorption ionization-time of flight mass spectrometry: a fundamental shift in the routine practice of clinical microbiology. Clin Microbiol Rev 2013; 26: 547603. doi: 10.1128/CMR.00072-12. 
6.- Deak E, Charlton C L, Bobenchik A M, Miller S A, Pollett S, McHardy I H, et al. Comparison of the Vitek MS and Bruker Microflex LT MALDI-TOF MS platforms for routine identification of commonly isolated bacteria and yeast in the clinical microbiology laboratory. Diagn Microbiol Infect Dis 2015; 81: 27-33. doi: 10.1016/j.diagmicrobio.2014.09.018.

7.- Criseo G, Scordino F, Romeo O. Current methods for identifying clinically important cryptic Candida species. J Microbiol Methods 2015; 111: 50-6. doi: 10.1016/j.mimet.2015.02.004

8.- Jamal W Y, Ahmad S, Khan Z U, Rotimi V O. Comparative evaluation of two matrix-assisted laser desorption/ionization time-of-flight mass spectrometry (MALDI-TOF) systems for the identification of clinically significant yeast. Int J Infect Dis 2014; 26:167-70. doi: 10.1016/j. ijid.2014.05.031.

9.- Westblade L F, Jenneman R, Branda J A, Bythrow M, Ferreraro M J, Garner O B, et al. Multicenter study evaluating the Vitek MS system for identification of medically important yeast. J Clin Microbiol 2013; 51: 2267-72. doi: 10.1128/JCM.00680-13.

10.- Porte L, García P, Braun S, Ulloa M, Lafourcade M, Montaña A, et al. Head-to-head comparison of Microflex LT and Vitek MS systems for routine identification of microorganisms by MALDI-TOF mass spectrometry in Chile. PLoS One 2017;12: e0177929. doi: 10.1371/journal.pone.0177929.

11.- Green M R, Sambrook J. Isolation of High-Molecular-Weight DNA Using Organic Solvents. Molecular Cloning: a Laboratory Manual. New York: CSH Press, 2012.

12.- White T J, Bruns T, Lee S, Taylor J. Amplification and direct sequencing of fungal ribosomal RNA genes for phylogenetics. In: Innis MA GD, Sninsky JJ, White TJ, ed. PCR Protocols: A Guide to Methods and Applications. San Diego: Academic Press, 1990; 315-22.

13.- Zhang L, Xiao M, Wang H, Gao R, Fan X, Brown M, et al. Yeast identification algorithm based on use of the Vitek MS system selectively supplemented with ribosomal DNA sequencing: proposal of a reference assay for invasive fungal surveillance programs in China. J Clin Microbiol 2014; 52: 572-7. doi: 10.1128/JCM.02543-13.

14.- Landis J R, Koch G G. The measurement of observer agreement for categorical data. Biometrics 1977; 33: 159-74.

15.- Falci D R, Pasqualotto A C. Clinical mycology in Latin America and the Caribbean: A snapshot of diagnostic and therapeutic capabilities. Mycoses 2019; 62: 368-73. doi: 10.1111/myc. 12890.

16.- Relloso M S, Nievas J, Santiago-Mares T, Farquharson V, Mujica M T, Romano V, et al. Evaluación de la espectrometría de masas: MALDITOF MS para la identificación rápida y confiable de levaduras. Rev Arg Microbiol 2015; 43:103-7. doi: 10.1016/j.ram.2015.02.004.

17.- Kassim A, Pflüger V, Premji Z, Daubenberger C, Revathi G. Comparison of biomarker based Matrix Assisted Laser Desorption Ionization-Time of Flight Mass Spectrometry (MALDI-TOF MS) and conventional methods in the identification of clinically relevant bacteria and yeast. BMC Microbiol 2017; 17: 128. doi: 10.1186/s12866-017-1037-z.

18.- Girard V, Mailler S, Chetry M, Vidal C, Durand G, van Belkum A, et al. Identification and typing of the emerging pathogen Candida auris by matrix-assisted laser desorption ionisation time of flight mass spectrometry. Mycoses 2016; 59: 535-8. doi: 10.1111/myc.12519.

19.- Ferreira L, Sánchez-Juanes F, Vega S, González M, García M I, Rodríguez $\mathrm{S}$, et al. Identification of fungal clinical isolates by matrix-assisted laser desorption ionization-time-of-flight mass spectrometry. Rev Esp Qumioter 2013; 26: 193-7.

20.- Colombo A L, Padovan A C, Chaves G M. Current knowledge of Trichosporon spp. and Trichosporonosis. Clin Microbiol Rev 2011; 24:682700. doi: 10.1128/CMR.00003-11. 